\title{
A NOTE ON CONVEX FUNCTIONS ${ }^{1}$
}

\section{BERNARD FRIEDMAN}

It is well known that, just from the definition of convexity, a function of one variable which is convex must also be absolutely continuous. It was not known whether this property would generalize to convex functions of more than one variable. In this note there is constructed a convex function of two variables which is not absolutely continuous.

Carathéodory's definition of absolute continuity [1] for functions of two variables may be stated as follows:

Let $f(x, y)$ be any functions of two variables and $S$ the square with the vertices

$$
(x-h, y-h), \quad(x-h, y+h), \quad(x+h, y-h), \quad(x+h, y+h) \text {. }
$$

Let $I(S)$ be the following interval function

$$
\begin{aligned}
I(S)= & f(x+h, y+h)+f(x-h, y-h) \\
& -f(x+h, y-h)-f(x-h, y+h) .
\end{aligned}
$$

Then $f(x, y)$ is said to be absolutely continuous in a two-dimensional region $R$ if $f(x, y)$ is absolutely continuous in each variable separately and if also $I(S)$ is an absolutely continuous function of $S$. Carathéodory [2] proves the absolute continuity of $f(x, y)$ is a necessary and sufficient condition that there exist functions $g(x), h(y)$ and $k(x, y)$ such that

$$
f(x, y)=\int_{0}^{x} g(x) d x+\int_{0}^{y} h(y) d y+\int_{0}^{x} \int_{0}^{y} k(x, y) d x d y+C .
$$

The counterexample of this note will be constructed from the integral of Cantor's middle third function which can be defined as follows:

$$
\begin{array}{rlrl}
m(0) & =0, & m(1) & =1, \\
m(x) & =1 / 2, & & 1 / 3 \leqq x \leqq 2 / 3, \\
m(x) & =1 / 4, & 1 / 9 \leqq x \leqq 2 / 9, \\
m(x) & =3 / 4, & 7 / 8 \leqq x \leqq 8 / 9, \cdots .
\end{array}
$$

It is well known that $m(x)$ is a continuous monotonically increasing function whose derivative is zero almost everywhere. Obviously $m(x)$

1 Presented to the Society, April 8, 1938. 
is not absolutely continuous and not convex, but $f(x)=\int_{0}^{x} m(t) d t$ will be both convex and absolutely continuous. $f(x)$ is convex since its derivative $m(x)$ is everywhere non-decreasing.

It is to be noticed that if $f(x)$ is a convex function of $x$, then $f(x, y) \equiv f(x+y)$ is a convex function of two variables $x$ and $y$.

However if $f(x, y)$ were absolutely continuous in the two variables $x$ and $y$, by Carathéodory's theorem it could be represented as

$$
f(x+y)=\int_{0}^{x} g(x) d x+\int_{0}^{y} h(y) d y+\int_{0}^{x} \int_{0}^{y} k(x, y) d x d y+C .
$$

But differentiating with respect to $x$ it follows that

$$
f^{\prime}(x+y)=m(x+y)=g(x)+\int_{0}^{y} k(x, y) d y
$$

for almost all values of $x$. Let $x_{0}$ be some value of $x$ for which the equality holds. Then

$$
m\left(x_{0}+y\right)=g\left(x_{0}\right)+\int_{0}^{y} k\left(x_{0}, y\right) d y
$$

or $m\left(x_{0}+y\right)$ would be absolutely continuous, which is impossible.

Therefore $f(x+y)$ is a convex function of two variables which is not absolutely continuous.

It is quite easy to prove directly that $I(S)$ is not absolutely continuous. The direct proof, however, is uninteresting and since it requires some tedious computations, it will be omitted.

\section{BIBLIOGRAPHY}

1. Carathéodory, Vorlesungen itber Reele Funktionen, 1918, p. 653.

2. Carathéodory, loc. cit., p. 654.

UNIVERSITY OF WisCONSIN 